\title{
A Plane Equivalent Micro-truss Element for Reinforced Concrete Structures
}

\author{
Zhihang Xue ${ }^{1}$, Eddie Siu-shu Lam ${ }^{1}$ \\ ${ }^{1}$ The Hong Kong Polytechnic University \\ Hung Hom, Kowloon Hong Kong, China \\ zhihang.xue@connect.polyu.hk; cesslam@polyu.edu.hk
}

\begin{abstract}
Plane or solid elements are the most commonly used elements for finite element analysis (FEA), especially for predicting the behaviour of reinforced concrete structures in engineering practices. However, there are some limitations in the continuum model built with plane or solid elements. Traditional FEA with the continuum model is not only time consuming due to numerous degrees of freedom but also difficult to predict crack propagation. Properly replacing the continuum model with micro-truss elements in the analysis could be cost effective, especially for automatically generating the strut-and-tie model (STM), which is an ideal tool for analysis and design of the distributed region (D region). In this paper, a micro-truss element consisting of four nodes, using horizontal, vertical and diagonal members only hinged at both ends of the element is proposed to replace the plane elements. The equivalence formulas between the micro-truss element and the plane element are derived. The result was validated by comparing the solution of three cases modelled by Abaqus standard element. Moreover, the feasibility of combining with other types of element was studied by modelling a simply supported beam with reinforcements.
\end{abstract}

Keywords: Micro-truss element, Finite element analysis, Reinforced concrete structures, Strut-and-tie model.

\section{Introduction}

Finite element method is a powerful tool for studying the behaviour of reinforced concrete structures. Plane or solid elements are commonly employed for analysing reinforced concrete structures in engineering practices. As plane or solid elements generate numerous degrees of freedom, the traditional analysis consumes long computational time. In reinforced concrete simulation, the continuum models built with plane or solid elements may fail to predict crack propagation which is governed by discrete fracture mechanism, while plane or solid elements are based on continuum theory. Recent studies investigated methods to replace continuum model by truss model to simplify the analysis procedure. This method is based on the framework method proposed by Hrennikoff [1] for solving problem of elasticity using trusses. It is accurate and cost effective [2]. In Salem's study, a micro-truss model was considered as a generalization of strut-and-tie model (STM) which is an ideal tool for analyzing the distributed region (D region). In the last two decades, topology optimization method was widely used to automatically develop the STM [3], however, optimal topology of the D region modelled by solid element could fail to reflect the characteristics of force spreading [4].The emergency of micro-truss element provide an enhanced method for modelling and developing the optimal strut-and-tie model[5]. Further, the two-dimensional micro-truss element can be extended to three-dimensional [6], which is more challenging for determine an optimal STM with continuum model for concrete.

In this paper, a micro-truss element which has four nodes and comprises horizontal, vertical and diagonal members only hinged at both ends of the element was proposed to replace the plan element. The result was validated by comparing the solution of three cases modelled by plane element. Feasibility of combing with other type of elements was studied with modelling a simply supported beam with reinforcement.

\section{Equivalent micro-truss element to plane stress element}

A micro-truss element, consisting of four nodes and six truss elements comprising two types of truss, is proposed as shown in Fig. 1. The trusses lie in the horizontal and vertical direction have the elastic modulus $\mathrm{E}_{1}$ and cross-section area $\mathrm{A}_{1}$, and the trusses lie in diagonal direction have the elastic modulus $\mathrm{E}_{2}$ and cross-section area $\mathrm{A}_{2}$. Two diagonal trusses are not connected but are crossed and unattached with each other. The micro-truss element provides more pathway for the load 
transfer in each internal node than the solid elements. Therefore, it is able to more accurately reflect the actual situations in a structure. However, there is a critical problem that still needs to be solved before the application of micro-truss element, which is how to replace the plane elements by the micro-truss element. An equivalent method is proposed to replace plane elements based on the equivalent deformation.

The idea of the deformation equivalent is to determine the stiffness of the above mentioned two types of trusses by equalling the deformation in compression or tension and deformation in pure shear respectively. The displacement equivalence ensures the performance of the micro-truss element is identical to the four-node plane element, which is a crucial point in modelling reinforced concrete structures. It can be assumed that normal stress and shear stress distribute uniformly. As shown in Fig. 2(a), it is assumed that normal stress $\sigma_{x}$ or $\sigma_{y}$ is normal to the x-axis or y-axis and shear stress $\tau_{x y}$ in the plane. For a micro-truss element in tension, the equivalent nodal force applied is shown in Fig. 2(b).

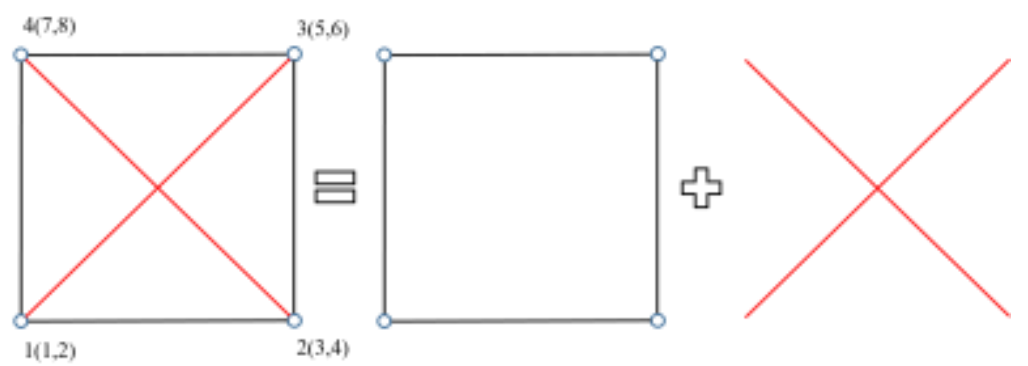

Fig. 1: Proposed micro-truss element.

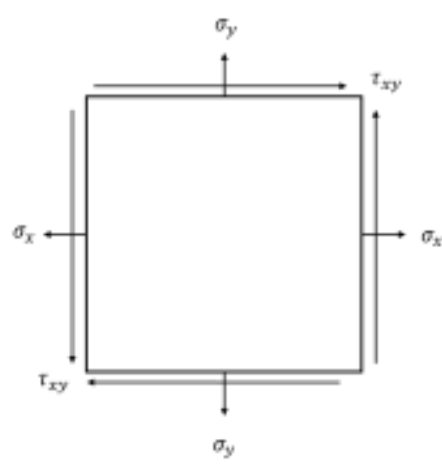

(a) Plane element

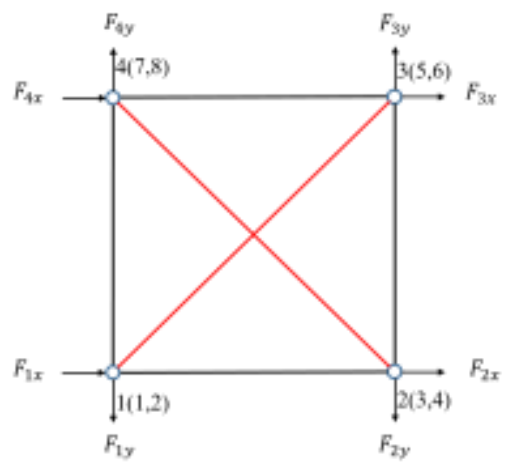

(b) Micro-truss element

Fig. 2: Load equivalent relationship of equivalent truss element and plane solid element.

The deformation mode of plane element in tension or compression or shear is shown in Fig. 3, and it could be solved according to Equations of (1) and (2)

$$
\begin{aligned}
& \Delta_{1, s}=\frac{\sigma_{x} L}{E} \\
& \Delta_{2, s}=\frac{\tau_{x y} L}{G}
\end{aligned}
$$


where, $\sigma_{\mathrm{x}}$, and $\tau_{\mathrm{xy}}$ are the stress components; $\mathrm{L}$ is the dimension of plane element; $\mathrm{E}$ and $\mathrm{G}$ are elastic modulus and shear modulus of material, respectively. The micro-truss element performs a similar deformation pattern under the equivalent nodal force condition as shown in Fig. 4. The deformation can be solved by MATLAB Programming and expressed as follow:

$$
\begin{gathered}
\Delta_{1, t}=\frac{\sigma_{x} L\left(4 A_{1}^{2} E_{1}^{2}+A_{2}^{2} E_{2}^{2}+2 \sqrt{2} A_{1} A_{2} E_{1} E_{2}\right)}{\left(4 A_{1} E_{1}+\sqrt{2} A_{2} E_{2}\right)\left(2 A_{1}^{2} K_{1}^{2}+\sqrt{2} A_{1} A_{2} E_{1} E_{2}\right)} \\
\Delta_{2, t}=\frac{\left(\tau_{x y} L\right)\left(4 A_{1} E_{1}+\sqrt{2} A_{2} E_{2}^{2}\right)}{\sqrt{2} A_{2}^{3} E_{2}^{3}+8 A_{1} A_{2}^{2} E_{1} E_{2}^{2}+8 \sqrt{2} A_{1}^{2} A_{2} E_{1}^{2} E_{2}}
\end{gathered}
$$

where $E_{1}$ and $E_{2}$ are elastic modulus of different trusses. As mentioned previously, the displacement equivalence mode can be described as

$$
\begin{aligned}
& \Delta_{1, s}=\Delta_{1, t} \\
& \Delta_{2, s}=\Delta_{2, t}
\end{aligned}
$$

Elastic modulus of different trusses in micro-truss element can be determined as follows:

$$
\begin{gathered}
E_{1}=\frac{E L-2 G L+2 L \sqrt{\frac{E^{2}}{4}+G^{2}}}{4 A_{1}} \\
E_{2}=\frac{\sqrt{2} G L}{A_{2}}
\end{gathered}
$$

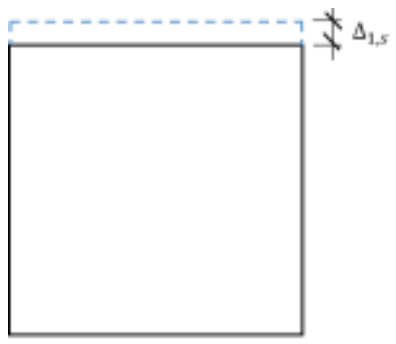

Fig. 3: Deformation modes of a plane element.

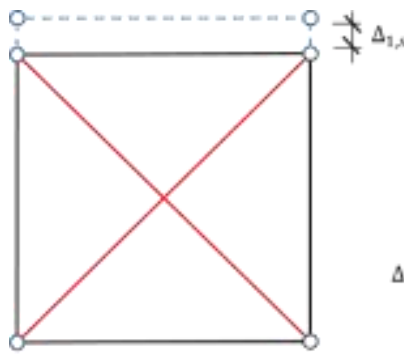

Fig. 4: Deformation modes of a micro-truss element.

The global stiffness matrix of each truss element can be formulated directly by assuming unit displacement in the global direction. Once the individual stiffness of each member is determined, the global stiffness matrix can be assembled. 


\section{Validation of micro-truss element}

Validation of micro-truss element was performed in three cases as shown in Fig. 5, including cantilever beam, simply supported beam with and without reinforcement. It aims to investigate the accuracy and convergence of the proposed microtruss element, also to study its compatibility with other type of elements. The test results are mainly focused on the deflection of beams. All of them are compared with those modelled with Abaqus standard element (CPS4) under identical loading and boundary conditions.
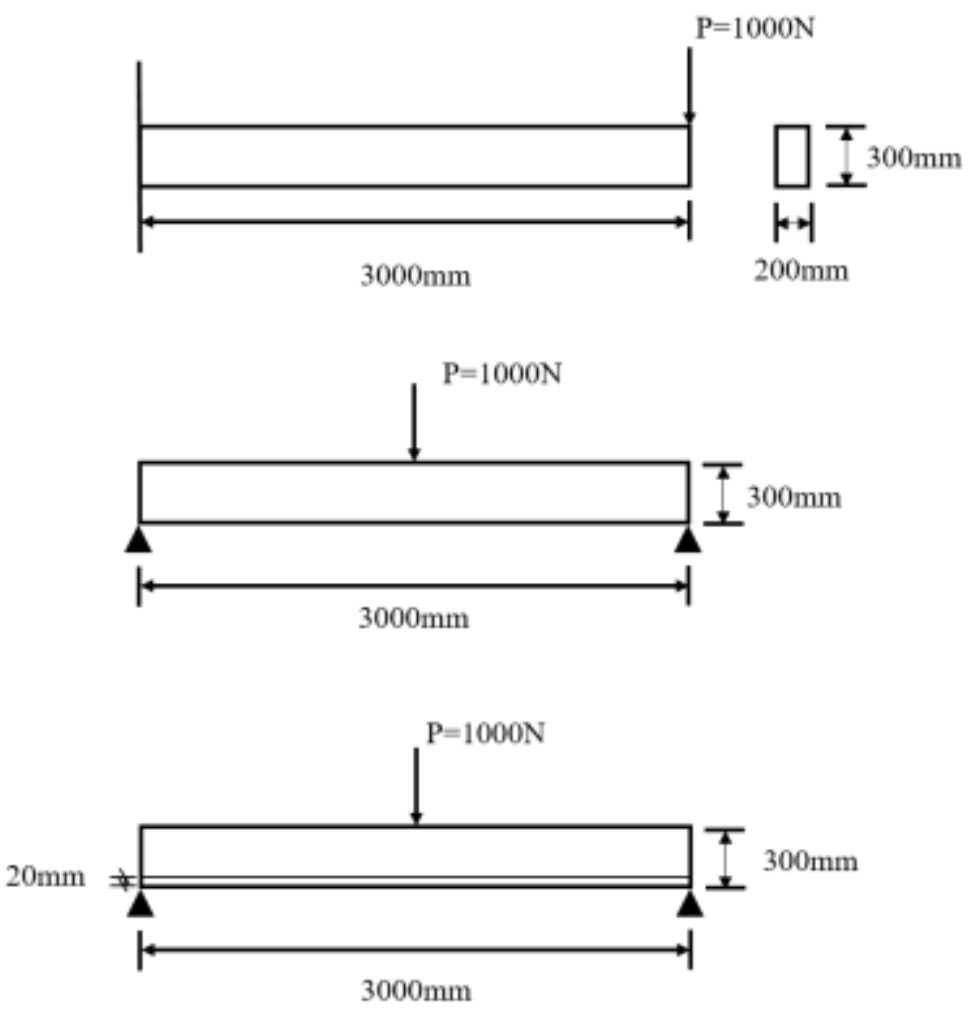

Fig. 5: Test cases for micro-truss element.

\subsection{Testing of cantilever}

A cantilever under a point load at its free end is a classic test used to assess the behaviour of a given finite element. The beam is $3000 \mathrm{~mm}$ in length, $300 \mathrm{~mm}$ in depth and $200 \mathrm{~mm}$ in width subjected to a concentrated load of $1000 \mathrm{~N}$ at the free end. It has an elastic modulus $\mathrm{E}$ of $30 \mathrm{GPa}$ and a Poisson's ratio of $v 0.3$. Four discretization using micro-truss element, $50 \times 50$ $\mathrm{mm}, 30 \times 30 \mathrm{~mm}, 20 \times 20 \mathrm{~mm}, 10 \times 10 \mathrm{~mm}$, have been tested to investigate the convergence of the element; and also to study the accuracy. Using Equations (7) and (8), stiffness of truss element can be formulated by K=EA/L. Subsequently the global stiffness of micro-truss element can be determined. Results of vertical deflection at free end are compared with the solutions from FEA which is modelled with the Abaqus standard element (CSP4). Details of beam modelled with Abaqus standard element are same with that of modelled with the proposed micro-truss element.

Deflection curves with the coarse mesh $(50 \times 50)$ are plotted in Fig. 6. In despite of using different element types, deflection of beam modelled by the micro-truss elements agrees well with that of the Abaqus standard element (CSP4). Furthermore, both of deflection curves remain largely the same as the analytical solutions. In detail, there is a maximum error of 3.0\% between the micro-truss element results and the analytical solutions. Fig. 7 shows the deflection of free end 
modelled with different mesh strategies. It can be seen that the relative error between micro-truss element and Abaqus standard element is small and it decreases quickly with increasing in element number. The solution is closed to the theoretical prediction value with the element size about 1/10 height of beam.

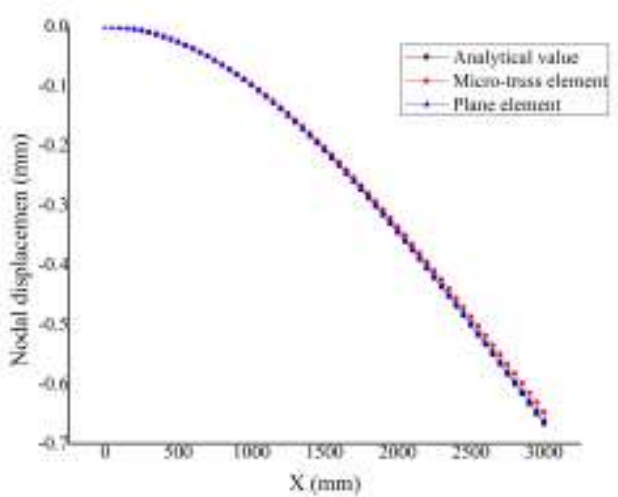

Fig. 6: The deflection curve of cantilever.

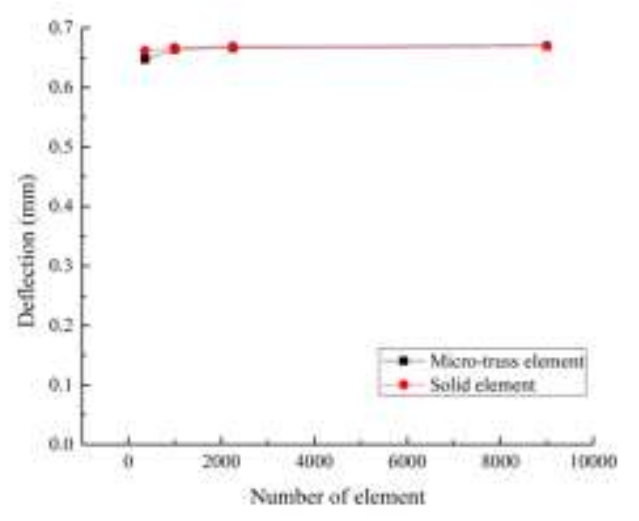

Fig. 7: Convergence of micro-truss element and solid element.

\subsection{Testing of simply supported beam}

A simply supported beam loaded at the mid-span was used as another example for testing the proposed micro-truss element. The beam is of $3000 \mathrm{~mm}$ in length, and $300 \mathrm{~mm}$ in depth subjected to a concentrated force of $1000 \mathrm{~N}$ in mid-pan. It has an elastic modulus E of 30GPa and Poisson's ratio $v$ of 0.3 . The simply supported beam is used to test the effectiveness of the micro-truss element in different structures with different element sizes of $50 \times 50 \mathrm{~mm}, 30 \times 30 \mathrm{~mm}, 20 \times 20 \mathrm{~mm}, 10 \times 10$ $\mathrm{mm}$. Results of the vertical deflection at the mid-span are compared with the solutions from the FEA method which was modelled with the Abaqus standard element (CPS4). Details of beam and load conditions are identical to that of modelling to micro-truss element.

The deflection curves of the beam with the coarse mesh $(50 \times 50)$ are plotted in Fig. 8. It can be seen from the figure that the deflection curve of the beam modelled with the micro-truss elements agree well with that of the Abaqus standard element (CSP4). Both deflection curves remain similar to the analytical solutions. Moreover, the error between the micro-truss element results, plane element results and the analytical solutions is small. As the number of elements increases, it is observed that the relative error reduces. With the element size close to $1 / 15$ height of beam, the analysis result can be accurately obtained. The deflection curve of beam modelled by the micro-truss element is close to that of Abaqus standard element as shown in Fig. 9. 


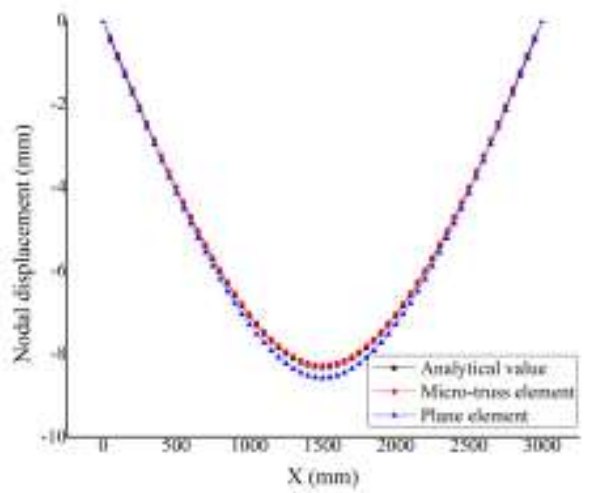

Fig. 8: The deflection curves of the simply supported beam.

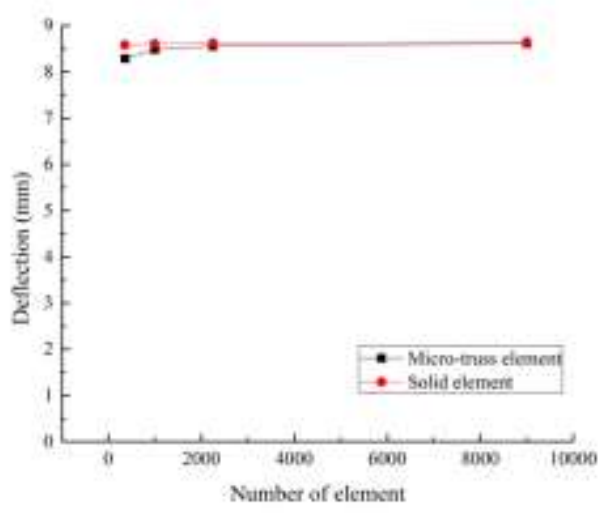

Fig. 9: Convergence of micro-truss element and solid element.

\subsection{Testing of simply supported beam with reinforcement}

The feasibility of the micro-truss element combing with other types of element is tested. The above mentioned simply supported beam with reinforcement at the bottom of the section is modelled with micro-truss element at the size of $20 \times 20$ $\mathrm{mm}$ for concrete and Abaqus standard element (T2D2) for reinforcement. The result is compared with concrete modelled by Abaqus standard element (CSP4) and reinforcement with truss element (T2D2). Details of beam and loading condition are the same as the above mentioned simply supported beam as shown in Fig. 5. The mid-span deflection for beam modelled with micro-truss element (i.e. $8.24 \mathrm{~mm}$ ) agrees well with that modelled with Abaqus standard element (e.g. $8.22 \mathrm{~mm}$ ). It indicates that the user-defined element can be combined with Abaqus standard element and performs reasonably with high accuracy. Fig. 11(a) shows the contour of deformation predicted by Abaqus standard elements. As shown in Fig. 11(b), reinforcement modelled with Abaqus standard element can be displaced, while the concrete modelled with the used-defined element cannot be presented. Deformation can be visualized by overlapping a dummy element with an almost vanishing material stiffness. This can be achieved using the subroutine UVARM, which is called at the end of every increment at every integration point of the dummy element mesh to generate element output.

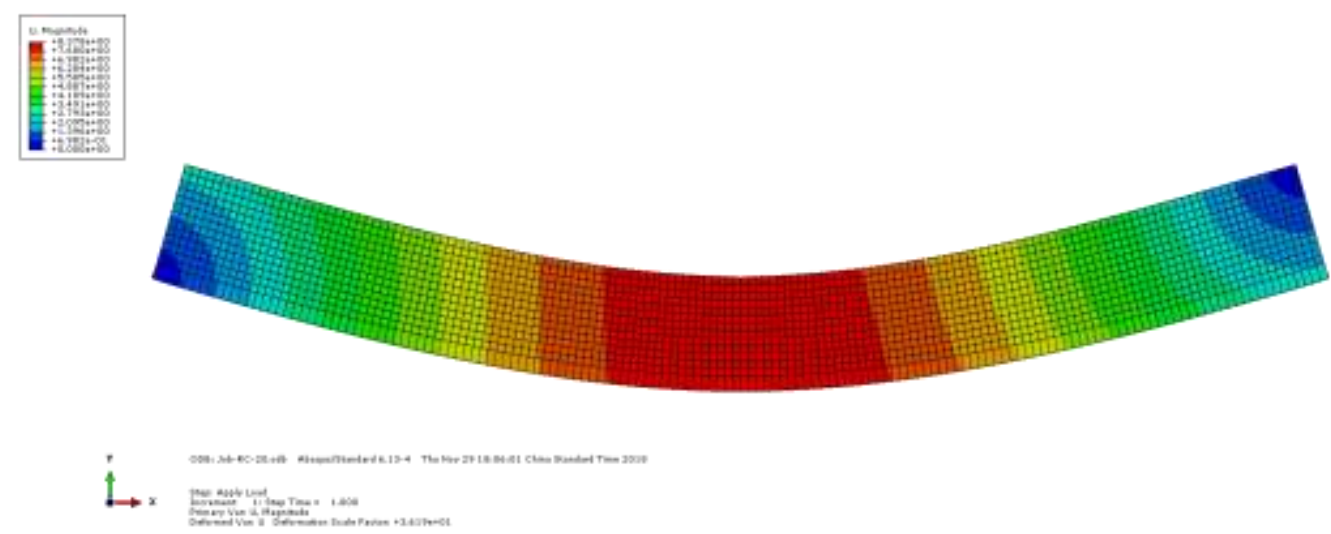

Fig. 10(a): Contours of deformed shape modelled with plane stress element. 


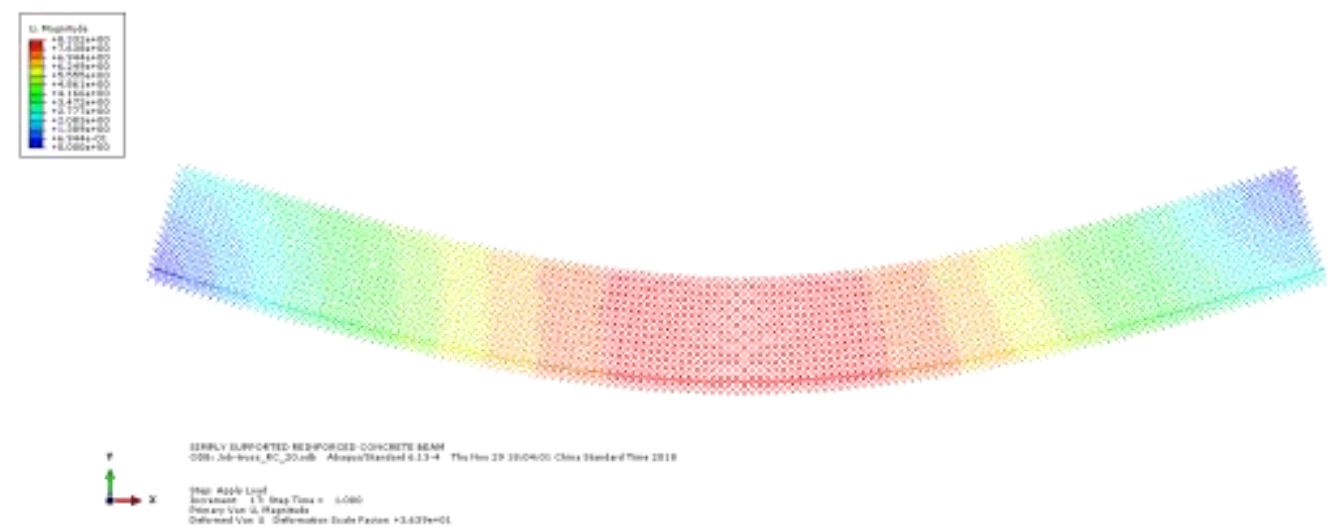

Fig. 10(b): Contours of deformed shape modelled with micro-truss element.

\section{Conclusion}

In this study, a micro-truss element has been proposed based on equivalent the deformation between the micro-truss element and plane element. The proposed element has been tested in different situations, including cantilever, simply supported beam with and without reinforcement to investigate its accuracy and convergence and also the feasibility of combing with other types of element. The simulation results are compared with the Abaqus standard element. The following conclusions can be drawn within the present study.

1) Performance of the proposed micro-truss element is closed to that obtained using as plane element. Only two of the properties related to plane element including elastic modulus E, shear modulus G, and Poisson ratio $v$ can be equivalent to micro-truss element. This may lead to a slightly error in lateral deformation. Another equivalent method based on energy will be studied in the future. Shear modulus of diagonal members to resist shear stress method can be equivalent to the stiffness of diagonal truss member. Thus, the performance of two elements is the same when elements are subjected to pure shear condition.

2) Accuracy and convergence of micro-truss element are tested by cantilever, simply supported beam with different sizes of element. The results in terms of deflection are compared with that of modelled with Abaqus standard element. The relative error of deflection modelled with two types of element decreased quickly with increasing element number, and the solution agree with the theoretical value with the element size about 1/10 to 1/15 height of the beam. It can be concluded that micro-truss element can well represent the plane element. The result of modeling simply supported beam with reinforcement shows that the micro-truss element can also be jointly used with other type of element with high accuracy.

\section{Acknowledgements}

The authors wish to express their gratitude and sincere appreciation to the financial support from The Hong Kong Polytechnic University. The author would like to thank Mr. Yuming Xu from National Cheng Kung University for his contribution to this study.

\section{References}

[1] A. Hrennikoff, "Solution of problems of elasticity by the framework method," J. appl. Mech., 1941.

[2] H. M. Salem, "The micro truss model: an innovative rational design approach for reinforced concrete," Journal of Advanced Concrete Technology, vol. 2, no. 1, pp. 77-87, 2004.

[3] Q. Q. Liang, B. Uy, and G. P. Steven, "Performance-based optimization for strut-tie modeling of structural concrete," Journal of Structural Engineering, vol. 128, no. 6, pp. 815-823, 2002.

[4] Y. M. Xie and G. P. Steven, "A simple evolutionary procedure for structural optimization," Computers \& Structures, vol. 49, no. 5, pp. 885-896, 1993. 
[5] J. Zhong, L. Wang, Y. Li, and M. Zhou, "A practical approach for generating the strut-and-tie models of anchorage zones," Journal of Bridge Engineering, vol. 22, no. 4, pp. 04016134, 2016.

[6] J. Zhong, L. Wang, M. Zhou, and Y. Li, "New Method for Generating Strut-and-Tie Models of Three-Dimensional Concrete Anchorage Zones and Box Girders," Journal of Bridge Engineering, vol. 22, no. 8, pp 04017047, 2017. 